\title{
Consecutive Jailed- and Kissing-Corsair Technique: Side Branch Protection and Dilation during Stent Implantation
}

\author{
Jungho Choi, Byeong-Keuk Kim, Sung-Jin Hong, Myeong-Ki Hong, and Yangsoo Jang \\ Division of Cardiology, Department of Internal Medicine, Severance Cardiovascular Hospital, Yonsei University College of Medicine, Seoul, Korea.
}

\begin{abstract}
The primary concern in percutaneous coronary intervention for bifurcation lesions is occlusion of a side branch after stenting of a main branch, especially in high-risk patients. We describe a novel technique, consecutive jailed- and kissing-Corsair technique, using a Corsair microcatheter for protection of side branches in bifurcation lesions.
\end{abstract}

Key Words: Percutaneous coronary interventions, bifurcation, stent

\section{INTRODUCTION}

Side branch (SB) occlusion, one of the most common complications during bifurcation intervention, ${ }^{1}$ can lead to worse clinical outcomes, such as cardiac death and myocardial infarction. Thus, clinical guidelines emphasize the importance of prevention of SB occlusion during bifurcation percutaneous coronary intervention (PCI) ${ }^{2,3}$ and recommend the provisional approach as a standard procedure. ${ }^{4,5}$ While this technique is relatively safe, there is risk of SB occlusion during PCI of bifurcation lesions. In our case series, we introduced a novel technique using a Corsair microcatheter. Patients of cases agreed and signed with informed consents.

\footnotetext{
Received: August 7, 2019 Revised: September 9, 2019

Accepted: September 14, 2019

Corresponding author: Byeong-Keuk Kim, MD, PhD, Division of Cardiology, Department of Internal Medicine, Severance Cardiovascular Hospital, Yonsei University College of Medicine, 50-1 Yonsei-ro, Seodaemun-gu, Seoul 03722, Korea. Tel: 82-2-2228-8457, Fax: 82-2-393-2041, E-mail: kimbk@yuhs.ac

-The authors have no potential conflicts of interest to disclose.

(c) Copyright: Yonsei University College of Medicine 2019

This is an Open Access article distributed under the terms of the Creative Commons Attribution Non-Commercial License (https://creativecommons.org/licenses/ by-nc/4.0) which permits unrestricted non-commercial use, distribution, and reproduction in any medium, provided the original work is properly cited.
}

\section{CASE REPORT}

\section{Case 1}

An 85-year-old female visited the emergency room due to severe dyspnea. The patient had a history of PCI at the mid-left anterior descending coronary artery (LAD) 2 months prior. Initial physical examination revealed bilateral coarse lung sounds and swelling of both legs with pitting edema. While initial electrocardiogram showed sinus rhythm, chest x-ray showed bilateral pulmonary congestion and cardiomegaly. Laboratory findings revealed elevation of troponin $\mathrm{T}(71 \mathrm{ng} / \mathrm{mL})$, suggestive of nonST-segment elevation myocardial infarction. Coronary angiography was performed and indicated severe stenosis at the obtuse marginal (OM) branch of the left circumferential artery (LCx) with a previous patent LAD stent (Xience Alpine, Abbott Vascular, Santa Clara, CA, USA) (Fig. 1A). For PCI of the OM branch, a 7-Fr XB 3.5 SH guide catheter (A\&A MD, Seongnam, Korea) was engaged via the femoral artery. Conventional guide wires (Runthrough wire, Terumo Medical Corporation, Somerset, NJ, USA; Pilot wire, Abbott Vascular) were inserted into both OM branches, the main target vessel, and LCx proper (SB), and then a Corsair microcatheter (Asahi Intecc, Nagoya, Japan) was inserted into the SB (LCx proper) for prevention of SB occlusion (Fig. 1B). After pre-dilation (2.5×14-mm semi-compliant balloon), a 3.0×28-mm everolimus-eluting stent (Synergy, Boston Scientific, Natick, MA, USA) was implanted into the OM branch under nominal inflation pressure (11 atm) with the Corsair catheter jailed at the SB (jailed-Corsair technique) (Fig. 1C, Supplementary Video 1, only online). After stenting, the jailed Corsair 
was removed by rotation, and rewiring was performed through the stent struts (Fig. 1D). The Corsair was recrossed to the SB under balloon anchoring $(2.5 \times 14 \mathrm{~mm})$ at the OM stent (Fig. 1E). After recrossing, high-pressure post-dilation (3.0×15 mm noncompliant balloon) was performed with the Corsair catheter at the SB (kissing-Corsair technique; Fig. 1F, Supplementary Video 2, only online). Final angiography revealed a well-expanded stent without dissection or flow limitation of the SB (Fig. 1G). After PCI, the patient was discharged without complications.

\section{Case 2}

A 60-year-old male presented with effort angina over the past 2 months. He was referred to our department for treatment of diffuse stenosis in the proximal-to-mid LAD and tight stenosis of the ostium of the diagonal branch (Fig. 2A). An 8-Fr XB 3.5 $\mathrm{SH}$ guiding catheter (A\&A MD) was engaged via the femoral artery. After wiring into the $\mathrm{LAD}, \mathrm{LCx}$, and diagonal branch, as well as pre-dilation of the LAD, a Corsair microcatheter (Asahi Intecc) was advanced to the diagonal branch for prevention of SB occlusion (Fig. 2B). With the Corsair jailed, a $3.5 \times 38-\mathrm{mm}$ everolimus-eluting stent (Xience Sierra, Abbott Vascular) was implanted at the LAD under nominal inflation pressure (14 atm) (jailed-Corsair technique; Fig. 2C). After stent implantation, the jailed Corsair was pulled back by rotation and rewired to the diagonal branch. Next, the Corsair microcatheter (Asahi Intecc) was recrossed through stent struts under balloon-anchoring at the LAD stent (Fig. 2D). With the Corsair microcatheter (Asahi Intecc) at the diagonal branch, a kissing-Corsair technique and proximal optimization were performed using a $3.5 \times 15-\mathrm{mm}$ non-compliant balloon (Fig. 2E). Final angiography exhibited no flow limitations of the diagonal branch and no residual stenosis in LAD stent (Fig. 2F). The patient was discharged without complications.

\section{DISCUSSION}

A jailed-wire technique or leaving a wire in the SB while implanting a stent into the main target vessel to prevent SB occlusion is preferred when the SB is not suitable for stenting or is clinically irrelevant and has an ostial or diffuse lesion, allowing protection of SB that may not require treatment but must remain patent. ${ }^{4,6}$ Although there is a risk of SB occlusion due to plaque or carinal shift following stent implantation, the jailed-balloon technique reduces such risks by maintaining the SB balloon while implanting the stent within the main vessel. ${ }^{7,8}$ However, this jailed-balloon technique can induce complications of balloon rupture or entrapment. More importantly, SB balloon inflation might cause dissection of the SB ostium, pose difficulties with rewiring to the SB, and lead to SB occlusion. To overcome these limitations, the jailed-Corsair technique was introduced using a Corsair microcatheter (Asahi Intecc), which has a tapered soft tip, a spiral
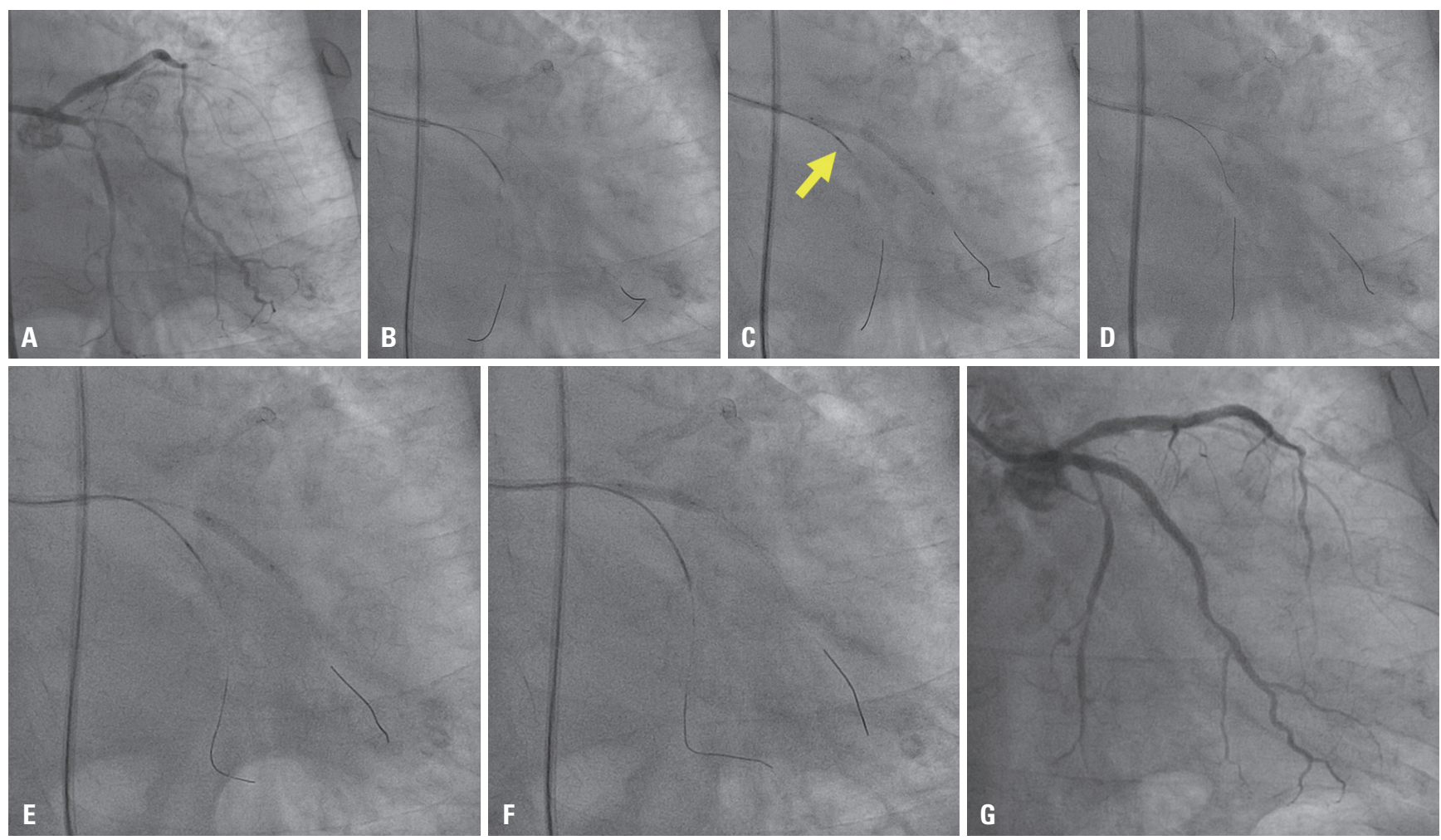

Fig. 1. (A) Pre-procedural angiography. (B) Insertion of the Corsair microcatheter into left circumferential artery (LCx) proper. (C) Jailed-Corsair technique. Stent implantation with the Corsair jailed (arrow). (D) Rewiring to the LCx proper through the stent struts. (E) Recrossing of the Corsair using anchor ballooning. (F) Kissing-Corsair technique. Post-dilation with Corsair at the LCx proper. (G) Final angiography. 

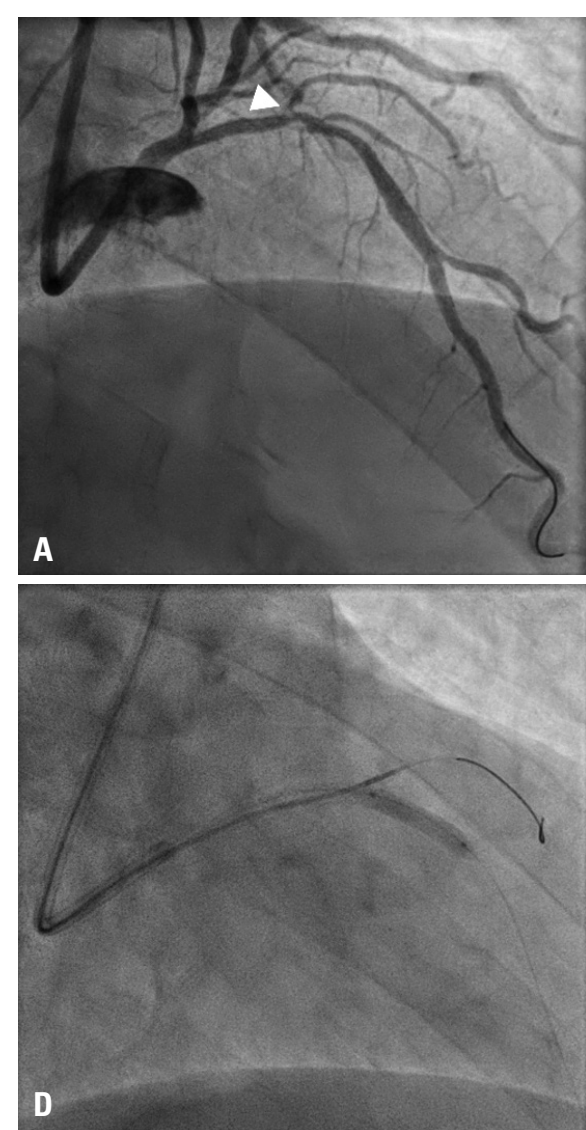
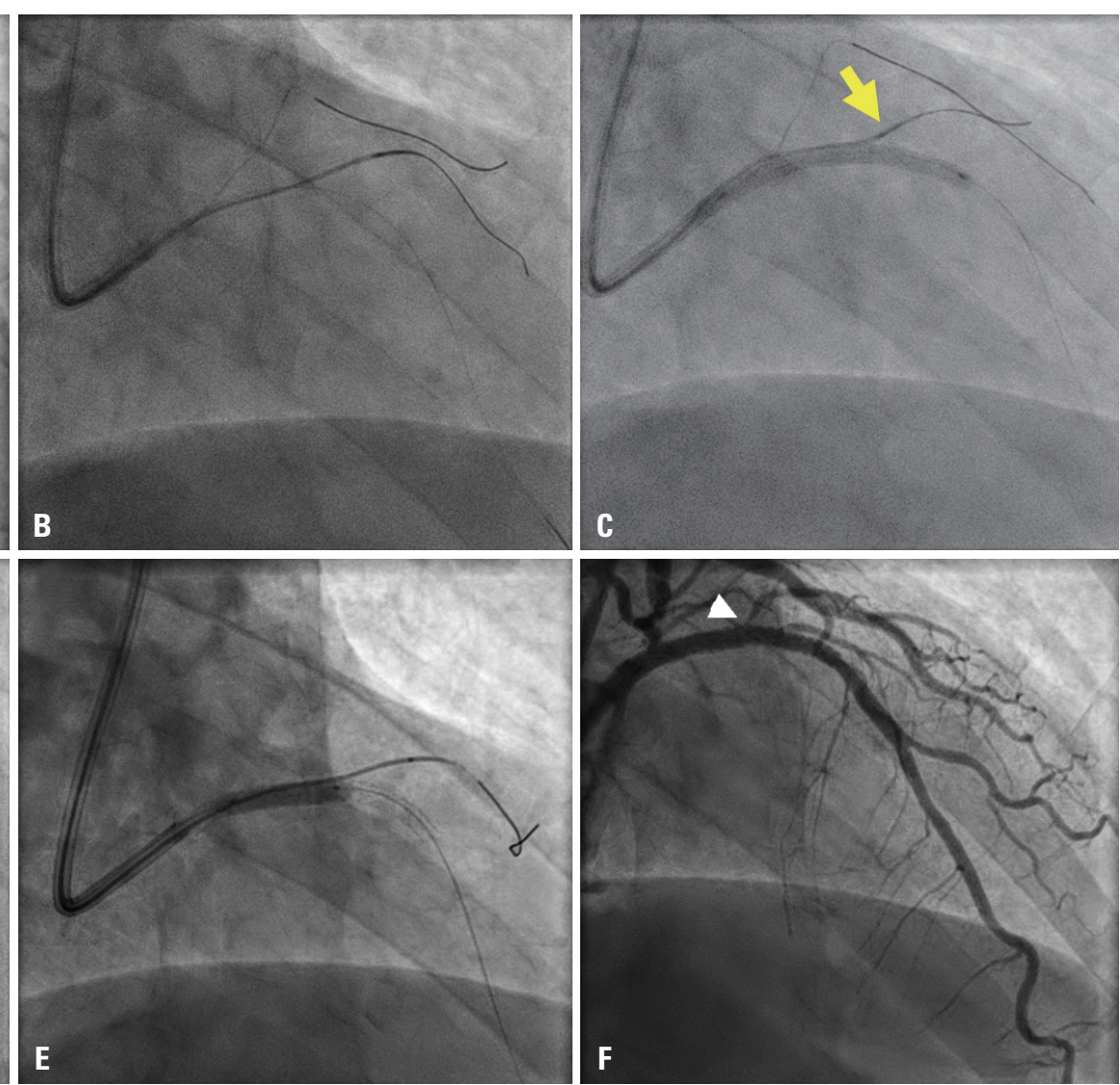

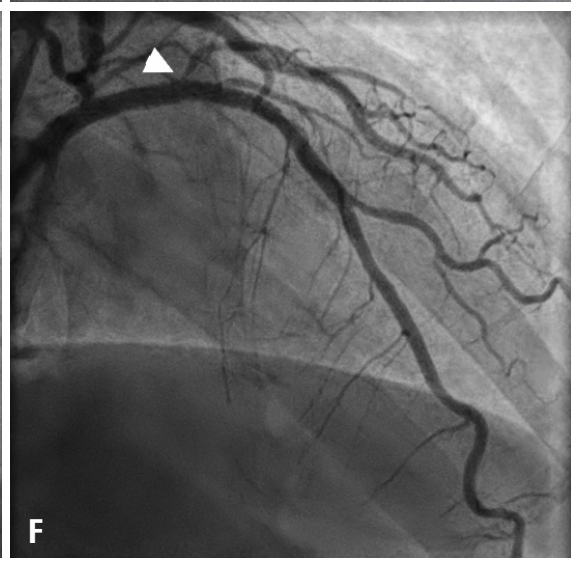

Fig. 2. (A) Pre-procedural angiography [tight stenosis of the ostium of the diagonal branch (arrowhead)]. (B) Insertion of Corsair into the diagonal branch. (C) Jailed-Corsair technique. Stent implantation with the Corsair jailed at the diagonal branch (arrow). (D) Recrossing of the Corsair using balloon anchoring. (E) Kissing-Corsair technique and proximal optimization. Post-dilation with the Corsair at the diagonal branch. (F) Final angiography with preserved side branch (arrowhead).

coil-shaft for transmitting rotation to the distal tip without twisting, and a hydrophilic coating to provide lubricity and maneuverability, showed better trackability, crossability, pushability, and strong back-up than other conventional microcatheters. ${ }^{9,10}$ Compared to the jailed-balloon technique, this technique poses a lower risk of dissection at the ostium of the SB and facilitates guide-wire recrossing to the SB. Based on the concept that the 1-mm Corsair shaft could serve as a small balloon, producing a quasi-kissing-balloon technique with minimized risks of SB occlusion, the kissing-Corsair technique was introduced as a treatment option for patients in whom small balloons cannot pass the SB or complex lesions in which a kissing balloon was not avilable. $^{11}$

The present cases demonstrated that our consecutive jailedand kissing-Corsair technique, facilitating SB protection and dilation before and after stent implantation, is an efficient, safe, and time-saving technique. This technique comprises a combined application of two conventional techniques, the jailed-Corsair and the kissing-Corsair techniques, for SB protection and treatment of bifurcation lesions. The consecutive jailed- and kissingCorsair technique can be applied in the PCI for bifurcation as follows: 1) the jailed-Corsair technique is used for SB protection; 2) after rewiring, the Corsair is advanced through the wire for minimal dilation of the SB ostium or the stenosis (balloon anchoring of the $\mathrm{MB}$ is useful for easy delivery of the Corsair); and 3) finally, the kissing-Corsair technique is performed using $\mathrm{MB}$ ballooning with the Corsair at the SB, and final proximal optimization using a high-pressure balloon is performed. This dual technique allows for widely opened stent struts and good SB scaffolding with protection of SBs. As demonstrated in our two cases, these two consecutive techniques seemed to minimize the complications associated with procedures on bifurcation lesions. The scheme of the consecutive jailed-Corsair technique and kissing-Corsair technique is shown in Fig. 3.

The jailed- and kissing-Corsair technique could pose risks of entrapment or tip transection. ${ }^{9}$ To prevent these, the tip of the Corsair catheter should be advanced distally and cross the SB ostium; moreover, stent-inflation pressure should not exceed nominal pressure. When removing the jailed Corsair, gentle pull-back with rotation of the catheter is definitely needed. For a safe procedure in heavy calcified bifurcation lesions, lesion modification using a non-compliant balloon, scoring balloon, or rotational atherectomy should be considered. ${ }^{12}$ In conclusion, a consecutive jailed- and kissing-Corsair technique using a Corsair microcatheter (Asahi Intecc) appears to prevent SB occlusion and open the SB ostium with minimal complications dur- 

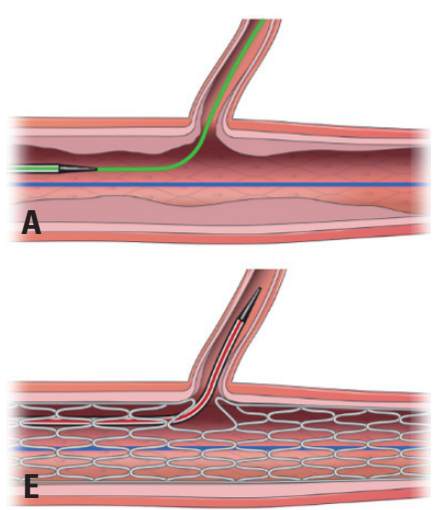
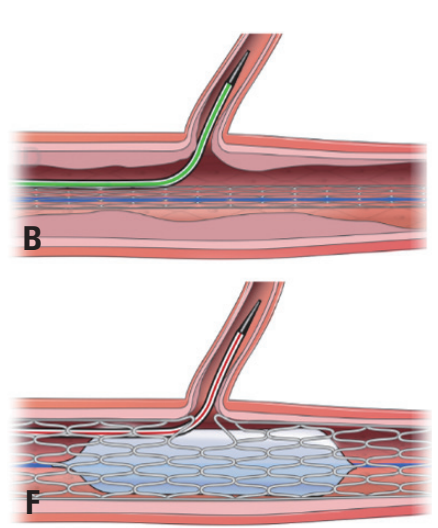
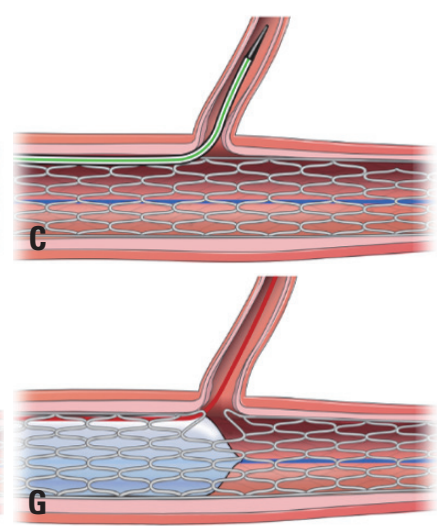
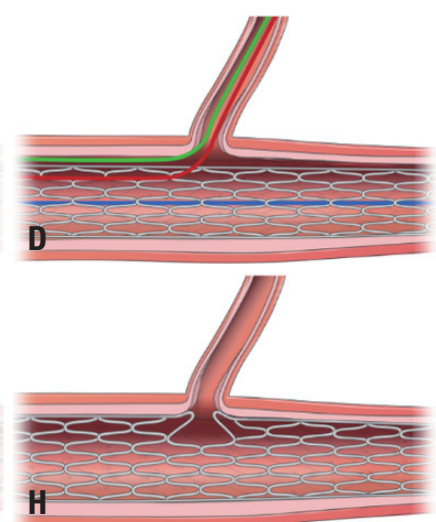

Fig. 3. (A) Wiring of the main branch (MB) (blue) and side branch (SB) (green). (B) Advancement of the Corsair to the SB. (C) Jailed-Corsair technique. Stent implantation (inflation with nominal pressure) with the Corsair jailed at the SB. (D) Removal of the jailed Corsair by rotation and rewiring to the SB (red wire). (E) Recrossing of the Corsair to the SB. (F) Kissing-Corsair technique; post-dilation of the MB with Corsair at the SB. (G) Proximal optimization using high-pressure post-dilation. $(\mathrm{H})$ Fully expanded MB stent with widely opened struts at the SB.

ing PCI of bifurcation lesions.

\section{SUPPLEMENTARY DATA}

Video 1. Jailed-Corsair technique. Stent implantation with the Corsair jailed at a side branch.

Video 2. Kissing-Corsair technique. Post-dilatation of the main branch with the Corsair at the side branch.

\section{ACKNOWLEDGEMENTS}

The authors thank Dong-Su Jang, Medical Illustration \& Design, a part of the Medical Research Support Services of Yonsei University College of Medicine, for help in designing the figures.

\section{AUTHOR CONTRIBUTIONS}

Conceptualization: Byeong-Keuk Kim. Data curation: Jungho Choi. Formal analysis: Sung-Jin Hong. Funding acquisition: Yangsoo Jang. Investigation: Jungho Choi. Methodology: Jungho Choi. Project administration: Myeong-Ki Hong. Resources: Byeong-Keuk Kim. Software: Jungho Choi. Supervision: Myeong-Ki Hong. Validation: SungJin Hong. Visualization: Jungho Choi. Writing_original draft: Jungho Choi. Writing—review \& editing: Byeong-Keuk Kim.

\section{ORCID iDs}

Jungho Choi https://orcid.org/0000-0001-9075-3222 Sung-Jin Hong htt://orcid.org/0000-0003-2493-066X $\begin{array}{ll}\text { Myeong-Ki Hong } & \text { https://orcid.org/0000-0003-4893-039X } \\ \text { Mtpid.org/0000-0002-2090-2031 }\end{array}$ Yangsoo Jang https://orcid.org/0000-0002-2169-3112

\section{REFERENCES}

1. Furukawa E, Hibi K, Kosuge M, Nakatogawa T, Toda N, Takamura $\mathrm{T}$, et al. Intravascular ultrasound predictors of side branch occlusion in bifurcation lesions after percutaneous coronary interven- tion. Circ J 2005;69:325-30.

2. Hahn JY, Chun WJ, Kim JH, Song YB, Oh JH, Koo BK, et al. Predictors and outcomes of side branch occlusion after main vessel stenting in coronary bifurcation lesions: results from the COBIS II Registry (COronary BIfurcation Stenting). J Am Coll Cardiol 2013;62: 1654-9.

3. Levine GN, Bates ER, Blankenship JC, Bailey SR, Bittl JA, Cercek B, et al. 2011 ACCF/AHA/SCAI Guideline for Percutaneous Coronary Intervention. A report of the American College of Cardiology Foundation/American Heart Association Task Force on Practice Guidelines and the Society for Cardiovascular Angiography and Interventions. J Am Coll Cardiol 2011;58:e44-122.

4. Lassen JF, Burzotta F, Banning AP, Lefèvre T, Darremont O, Hildick-Smith D, et al. Percutaneous coronary intervention for the left main stem and other bifurcation lesions: 12th consensus document from the European Bifurcation Club. EuroIntervention 2018;13:1540-53.

5. Sawaya FJ, Lefèvre T, Chevalier B, Garot P, Hovasse T, Morice MC, et al. Contemporary approach to coronary bifurcation lesion treatment. JACC Cardiovasc Interv 2016;9:1861-78.

6. Latib A, Colombo A. Bifurcation disease: what do we know, what should we do? JACC Cardiovasc Interv 2008;1:218-26.

7. Singh J, Patel Y, Depta JP, Mathews SJ, Cyrus T, Zajarias A, et al. A modified provisional stenting approach to coronary bifurcation lesions: clinical application of the "jailed-balloon technique". J Interv Cardiol 2012;25:289-96.

8. Burzotta F, Trani C, Sianos G. Jailed balloon protection: a new technique to avoid acute side-branch occlusion during provisional stenting of bifurcated lesions. Bench test report and first clinical experience. EuroIntervention 2010;5:809-13.

9. Numasawa Y, Sakakura K, Yamamoto K, Yamamoto S, Taniguchi Y, Fujita $\mathrm{H}$, et al. A novel side branch protection technique in coronary stent implantation: Jailed Corsair technique. Cardiovasc Revasc Med 2017;18:295-8.

10. Otsuka Y, Nakamura K, Saito T. Usefulness of the Corsair microcatheter for treatment of complex chronic total occlusion. J Invasive Cardiol 2012;24:E35-8.

11. Yoshida R, Takagi K, Morita Y, Morishima I. A novel bailout technique of jailed side branch dilatation following stent implantation: kissing-Corsair technique. JACC Cardiovasc Interv 2019;12:e53-4.

12. Sakakura K, Taniguchi Y, Matsumoto M, Wada H, Momomura S, Fujita H. How should we perform rotational atherectomy to an angulated calcified lesion? Int Heart J 2016;57:376-9. 